\title{
Anticoagulant therapy for non-ST- segment elevation acute coronary syndrome in China: A multi-center observational study
}

\author{
Xiao-huan Gong1, Jin-ming Yu${ }^{1}$, Yong Mao', Da-yi $\mathbf{H u}^{2}$ \\ ${ }^{1}$ Key Laboratory of Public Health Safety, Ministry of Education, Centre for Clinical Epidemiology, School of \\ Public Health, Fudan University, Shanghai 200032, China; \\ ${ }^{2}$ Heart Center, Peking University People's Hospital, Peking 100044, China
}

\section{ABSTRACT}

Objective: To assess the anticoagulant therapy for non-ST-segment elevation acute coronary syndrome (NSTE-ACS) in China and to offer the rationale for establishing reasonable strategies to improve the prognosis of NSTE-ACS. Methods: A total of 1,502 patients with NSTE-ACS were recruited from 28 third-grade hospitals distributed in 14 provinces and cities in China from December 2009 to December 2011. The strategies for diagnosis and treatment, decided by each hospital respectively, were used for further analysis and comparison of medication, percutaneous coronary intervention (PCI), and end points for efficacy and safety assessment at 9 and 30 days following PCI. Results: A lower incidence rate $(P<0.05)$ was noted for efficacy and safety in patients with unstable angina (UA) than those with non-ST-segment elevation myocardial infarction (NSTE-MI). The prescription rate of unfractionated heparin (UFH), low-molecular-weight heparin (LMWH), fondaparinux, $\mathrm{PCl}$, and single medication was $0.61 \%, 66.42 \%, 30.61 \%, 69.64 \%$, and $70.74 \%$, respectively. Conclusion: Compared with NSTE-MI, UA is featured with better prognosis, less severity, and different outcome. However, in clinical practice, the therapies for NSTE-MI and UA show no differences, which deserves great attention. In China, the most common anticoagulant therapies for NSTE-ACS are single medication, mainly based on LMWH and PCI.

Key words: acute coronary syndrome; anticoagulant; unstable angina; ST-segment elevation; myocardial infarction

Address for Correspondence: Jin-ming Yu,

Key Laboratory of Public Health Safety, Ministry of Education, Centre for Clinical Epidemiology, School of Public Health,

Fudan University, Shanghai 200032

China.Email: jmy@fudan.edu.cn

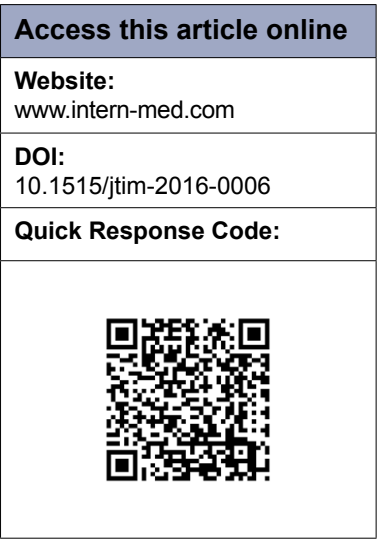

\section{INTRODUCTION}

Acute coronary syndrome (ACS) encompasses unstable angina (UA), non-ST-segment elevation myocardial infarction (NSTE$\mathrm{MI}$ ), and ST-segment elevation myocardial infarction (STE-MI). UA and NSTE-MI are classified as non-ST-segment elevation acute coronary syndrome (NSTE-ACS). Lifethreatening NSTE-ACS is a leading cause of emergency and hospitalization in the United States. ${ }^{[1]}$ It causes a lot of public expenditures. Its cost might account for a large proportion of worldwide health care expenditures in the future. ${ }^{[2]}$

The basic medicine for the treatment of NSTE-ACS is anticoagulants. The common anticoagulants are unfractionated heparin (UFH) and low-molecular-weight heparin
(LMWH), whose clinical efficacy has been proved by many clinical trials. A new anticoagulant, fondaparinux, with satisfactory efficacy and safety, may play a crucial role in the treatment of ACS. ${ }^{[3]}$ Based on the clinical data of 1,502 NSTE-ACS patients, the study aims to assess the anticoagulant therapy for NSTE-ACS in China, explore the severity of its two subtypes, reveal the problems in the treatment of NSTE-ACS, and offer the rationale for establishing reasonable strategies to improve the prognosis of NSTE-ACS.

\section{PATIENTS AND METHODS}

\section{Patients}

A total of 1,502 patients with NSTE-ACS were recruited from 28 third-grade hospitals distributed in 14 provinces and cities such as 
Peking, Tianjing, and Hangzhou in China from December 2009 to December 2011. Among them, aged over 21 (WHO diagnostic criteria) with the average age being $63.8 \pm 10.6$, 1,041 patients had UA and 461 NSTE-MI, of whom, 1,024 were male, accounting for $68.2 \%$.

\section{Methods}

The project is a multi-center observational study. The patients with NSTE-ACS, who met the inclusion criteria for the study, were recruited. The strategies for diagnosis and treatment were not stipulated or recommended by the project organizers, but decided by the researchers in each hospital respectively. The researchers were only encouraged to adopt the therapies recommended in the present guidelines for the management of NSTE-ACS. The medicines and percutaneous coronary intervention (PCI) that the subjects received were recorded.

For the subjects who had better use of UFH and (or) LMWH, the type of medicine, daily dosage, and course were decided by the researchers based on the clinical manifestations. For those who had better use of fondaparinux, the medicine was administered by hypodermic injection ( $2.5 \mathrm{mg} \mathrm{qd}$ ) for 8 days or until the hospital stay ended. The primary end points for efficacy (death, MI, refractory ischemia, stroke) and safety (hemorrhage) at 9 days and the secondary end points at 30 days following PCI were analyzed.

\section{Statistical Analysis}

The corpus was established using Epidata 3.1. The data were analyzed using IBM SPSS 19.0 and Stata 10.0 software. The count data were expressed by cases and percentage $(\%)$ and the normally distributed measurement data by mean $\pm \mathrm{SD}$. The measurement data were analyzed by t-test, analysis of variance, Wilcoxon rank sum test, and Kruskal-Wallis test. The count data were analyzed by $\mathrm{X}^{2}$ test or Fisher's exact test. The differences were considered significant when $P<0.05$.

\section{RESULTS}

\section{Comparison between UA and NSTE-MI}

The prescription rate of single medication (restricted to anticoagulants) among patients with UA was higher than that among those with NSTE-MI, and the prescription rate of PCI among patients with UA was slightly higher than that among patients with NSTE-MI; yet, the differences were not statistically significant $(P>0.05)$.

The pretreatment hemorrhagic risk appraisal score of the UA group was lower than that of the NSTE-MI group $(P<0.05)$. Lower incidence rates $(P<0.05)$ were noted for the primary efficacy and safety end points in the UA group than in the NSTE-MI group (Table 1).

\section{USE OF ANTICOAGULANTS}

Among NSTE-ACS patients who received medication, the prescription rate of UFH was less than $1 \%$, that of $\mathrm{LMWH}$ was more than $60 \%$, that of fondaparinux was almost $33 \%$, and that of other medications rather than anticoagulants was $2.36 \%$ (Table 2 ).

\section{Interventional and conservative therapies}

Among NSTE-ACS patients, 456 underwent conservative therapy, accounting for $30.36 \%$ (95\% CI: $28.04 \%$ $32.75 \%$ ), and 1,046 interventional therapy, accounting for 69.64\% (95\% CI: $67.25 \% \sim 71.96 \%$ ).

\begin{tabular}{|c|c|c|c|c|c|}
\hline & \multirow{2}{*}{$\begin{array}{l}\text { Hemorrhagic } \\
\text { risk appraisal } \\
\text { score }\end{array}$} & \multicolumn{2}{|c|}{ Medication [case (\%)] } & \multirow[t]{2}{*}{$\mathrm{PCl}[$ case $(\%)]$} & \multirow{2}{*}{$\begin{array}{l}\text { Primary efficacy Primary safety } \\
\text { end points [case end points [case } \\
(\%)] \\
(\%)]\end{array}$} \\
\hline & & Single & Combined & & \\
\hline UA $(1,041$ cases $)$ & $27.51 \pm 12.81$ & $734(72.10)$ & $284(27.90)$ & $725(70.32)$ & $1(0.10) \quad 2(0.19)$ \\
\hline NSTE-MI (461 cases) & $29.83 \pm 16.10$ & $308(67.84)$ & $146(32.16)$ & $304(67.26)$ & $4(0.88)$ \\
\hline$P$ value & 0.007 & 0.097 & & 0.239 & 0.033 \\
\hline
\end{tabular}

\begin{tabular}{lll}
\hline Table 2: Use of anticoagulants & & \\
\hline & Cases & Rate (95\% Cl) \\
\hline UFH & 9 & $0.61(0.28 \sim 1.15)$ \\
LMWH & 983 & $66.42(63.95 \sim 68.82)$ \\
Enoxaparin & 296 & $20.00(18.00 \sim 22.13)$ \\
Nadroparin & 204 & $13.78(12.07 \sim 15.65)$ \\
Dalteparin & 141 & $9.53(8.08 \sim 11.14)$ \\
LMWH made in China & 342 & $23.11(20.98 \sim 25.34)$ \\
Fondaparinux & 453 & $30.61(28.27 \sim 33.03)$ \\
Non-anticoagulants & 35 & $2.36(1.65 \sim 3.27)$ \\
\hline
\end{tabular}




\section{Single and combined medications}

Among NSTE-ACS patients who received medication, 1,047 cases used single medication, accounting for $70.74 \%$ (95\% CI: $68.35 \% \sim 73.05 \%$ ), and 433 combined medication, accounting for $29.26 \%$ (95\% CI: $26.95 \%$ $31.65 \%$ ).

\section{DISCUSSION}

NSTE-ACS, composed of UA and NSTE-MI, is characterized by an imbalance between myocardial oxygen supply and demand. The most common cause is rapid coronary artery narrowing and myocardial perfusion reduction caused by a non-occluded thrombus that develops on a disrupted atherosclerotic plaque. ${ }^{[4]}$ The clinical manifestations of NSTE-MI are similar to those of UA; yet they are more severe and lasting. UA may develop to NSTE-MI or STE-MI. ${ }^{[5]}$

Compared with the NSTE-MI group, lower pretreatment hemorrhagic risk appraisal score and lower incidence rates for the primary efficacy and safety end points were found in the UA group. The differences were of statistic significance, indicating that compared with the NSTE-MI group, the UA group had lower hemorrhagic risk, higher safety, and better response to treatment. However, no obvious tendency was noted in the treatments (including single medication, combined medication, PCI, and conservative therapy) of UA and NSTE-MI, suggesting that in clinical practice, UA and NSTE-MI are treated similarly. UA and NSTE-MI differ in hemorrhagic risk, safety, and outcome; yet, the treatment of the two diseases did not show any differences, implying that the different types of NSTE-ACS are treated similarly and their differences in severity are neglected in clinical practice. To sum up, NSTE-MI is different from UA in prognosis and severity, but lacks specific treatment. Therefore, it is essential to classify NSTE-ACS timely, formulate early strategies based on risk stratification, and decide the specific therapies for different subtypes so as to improve the prognosis of NSTE-ACS, especially that of severe NSTE-MI. ${ }^{[6]}$

Since the different subtypes of NSTE-ACS are treated in the same way, the overall anticoagulant therapy for NSTEACS was assessed, regardless of the differences among the subtypes. The anticoagulant therapy is an important part of NSTE-ACS therapy. It is recommended for Class IA in ACC/AHA guideline, which should be started immediately after antiplatelet therapy. ${ }^{[1]}$ The common conventional anticoagulant agents for NSTE-ACS are UFH and LMWH. UFH has been applied widely in clinical practice and can be easily monitored, so it has been considered as an effective anticoagulant agent in 2002 guideline. ${ }^{[7]}$ Compared with UFH, LMWH (including dalteparin, enoxaparin, nadroparin etc.) is characterized with longer half-life, higher bioavailability, and easier administration. Severe bleeding has been found in patients treated with UFH or LMWH, while the differences are not significant. ${ }^{[8]} \mathrm{LMWH}$ might replace UFH in elective PCI. ${ }^{[9]}$ Fondaparinux, a new anticoagulant, has satisfactory efficacy and safety, which are similar to those of enoxaparin. ${ }^{[10]}$ OASIS 5 study has showed that compared with enoxaparin, fondaparinux is associated with a significantly reduced number of deaths in patients with NSTE-MI at 30 days $(17 \% ; P=0.02)$ and lower rate of major bleeding $(P<0.001)$, and also revealed differences between the two agents in long-term followup. ${ }^{[1]}$ Compared with conventional agents, fondaparinux is associated with lower rate of hemorrhagic risk, so it is likely to be a promising anticoagulant agent, especially for the patients with high bleeding risk.

According to GRACE report (2005) on the anticoagulant therapy for ACS in China, the rate of patients receiving UFH or LMWH is about $75 \%$ and that of LMWH is over $50 \%$, among which the rate of enoxaparin is around $50 \%$ and that of other $\mathrm{LMWH}$ is $30 \%$, indicating a high prescription rate of LMWH and no obvious tendency in application. ${ }^{[12]}$ In our study, the prescription rate of UFH and LMWH was $0.61 \%$ and $66.42 \%$, respectively, proving that the prescription rate of LMWH for NSTEACS in China is high and that of UFH is very low; that of fondaparinux is more than $30 \%$; that of bivalirudin is zero; no obvious tendency (9-24\%: LMWH made in China $>$ enoxaparin $>$ nadroparin $>$ dalteparin) is seen in the application of LMWH. Our study has shown the present anticoagulant therapy for NSTE-ACS in China: the prescription rate of $\mathrm{LMWH}$ is high, that of $\mathrm{UFH}$ is very low, and that of fondaparinux has increased. Compared with conventional anticoagulants, fondaparinux has better efficacy and higher safety, so it should be more widely applied, especially for patients with high hemorrhagic risk, for whom fondaparinux could significantly reduce bleeding risk, improve prognosis, and decrease death rate.

As for high risk NSTE-ACS patients, stents might stabilize ruptured plagues. ${ }^{[5]}$ The use of PCI at early stage could reduce long-term death rate and morbidity ${ }^{[13]}$ Our study has shown that compared with conservative therapy, the rate of PCI is high $(69.64 v$ s. $30.36 \%)$, higher than that of PCI in China in $2005(50 \%) \cdot{ }^{[14]}$ Generally, the high rate of PCI for NSTE-ACS suggests that early invasive treatment based on risk stratification has been accepted and applied in China. The clinicians should provide more care to the patients who have not received PCI, accounting for over $30 \%$.

Antiplatelets, often used with the anticoagulant therapy, can prevent the formation of thrombi and protect ischemic 
myocardium. The most common antiplatelets are aspirin and clopidogrel. Aspirin should be administered to each patient with UA and (or) NSTE-MI unless they have contraindications; clopidogrel can reduce the rate of MI and death in patients with angina by $53.2 \%{ }^{[5]}$ The combined use of the anticoagulant and antiplatelet therapies could prevent the formation of thrombi effectively, so the combined medication is recommended. In our study, the rate of single medication is significantly higher than that of combined medication $(70.74 \%$ vs. $29.26 \%)$. The lower rate of combined medication indicates that many clinicians in China are not aware of the efficacy of such medicine, so it should be known and used by more clinicians in the future.

NSTE-ACS affects health and happiness. We have compared the medication of its two subtypes, analyzed the efficacy and safety, PCI, and prognosis of the anticoagulant therapy for patients with NSTE-ACS, and provided the rationale for establishing specific and reasonable strategies to improve the prognosis of NSTE-ACS in China.

\section{Conflicts of Interest}

None declared.

\section{REFERENCES}

1. Wright RS, Anderson JL, Adams CD, Bridges CR, Casey DE Jr, Ettinger SM, et al. 2011 ACCF/AHA Focused Update Incorporated Into the ACC/ AHA 2007 Guidelines for the Management of Patients With Unstable Angina/Non-ST-Elevation Myocardial Infarction: a report of the American College of Cardiology Foundation/American Heart Association Task Force on Practice Guidelines developed in collaboration with the American Academy of Family Physicians, Society for Cardiovascular Angiography and Interventions, and the Society of Thoracic Surgeons. J Am Coll Cardiol 2011; 57: e215- e367.

2. Hu DY, Jia SQ, Wang JY. Acute Coronary Syndromes. [Translated from Acute Coronary Syndromes, Théroux P]. Peking: People’s Medical Publishing House, 2007: 9.
3. Song L, Yan HB. Application of fondaparinux in the treatment of acute coronary syndrome. Chin J Cardiol 2010; 38: 472-4.

4. Ma AQ, Hu DY. Cardiology. Peking: People's Medical Publishing House, 2005:304.

5. Chinese Society of Cardiology, editorial board of Chinese Journal of Cardiology. Diagnostic and therapeutic guidelines for the management of patients with unstable angina/non-ST-elevation myocardial infarction. Chin J Cardiol 2007; 35: 295-304.

6. Han YL. Individualized therapy - an orientation for the antithrombus therapy for coronary heart diseases. Med J Chin PLA 2008; 33: 921-2.

7. Shi XB. The anticoagulant therapy 2008. Paper collections from The $10^{\text {th }}$ South China International Congress of Cardiology, Guangzhou, 2008.

8. Zhai ZG, Wang C, Liu YM, Qin ZQ. Comparison of unfractionated heparin and low molecular weight heparin in pulmonary thromboembolism: meta-analysis. Acta Acad Med Sin 2004; 26: 221-6.

9. de Lemos JA, Blazing MA, Wiviott SD, Brady WE, White HD, Fox KA, et al. Enoxaparin versus unfractionated heparin in patients treated with tirofiban, aspirin and an early conservative initial management strategy: Results from the A phase of the A-to-Z trial. Eur Heart J 2004; 25: 168894.

10. Simoons ML, Bobbink IW, Boland J, Gardien M, Klootwijk P, Lensing $\mathrm{AW}$, et al. A dose-finding study of fondaparinux in patients with nonST-segment elevation acute coronary syndromes: the pentasaccharide in unstable angina (PENTUA) study. J Am Coll Cardiol 2004; 43: 2183-90.

11. Yusuf S, Mehta SR, Chrolavicius S, Afzal R, Pogue J, Granger CB, et al. Fifth Organization to Assess Strategies in Acute Ischemic Syndromes Investigators, Yusuf S, Mehta SR, et al. Comparison of fondaparinux and enoxaparin in acute coronary syndromes. N Engl J Med 2006; 354 : 1464-76.

12. Hu DY, Huo Y, Ma CS. Diagnostic and therapeutic guidelines for cardiovascular diseases. Peking: People’s Medical Publishing House, 2009: 229.

13. Lv SZ. Optimal therapeutic strategy in patients with non-ST-segment elevation acute coronary syndrome (NSTE-ACS). China Medical Herald 2010; 7: 5.

14. Multi-central Collaborative Group on Chinese Registry of Acute Coronary Events. Comparison of current clinical practice and guideline application in therapies of ACS: findings from the Multi-central Collaborative Group on Chinese Registry of Acute Coronary Events. Chin J Cardiol 2005; 33: 789-92.

How to cite this article: Gong X, Yu J, Mao Y, Hu D. Anticoagulant therapy for non-ST-segment elevation acute coronary syndrome in China: A multicenter observational study. J Transl Intern Med 2014;4:25-8. 